\title{
Verbesserter Nachweis einer Aspergillose bei CF-Patienten
}

\author{
Eine britische Arbeitsgruppe hat einen serologischen Marker \\ entdeckt, der bei Patienten mit zystischer Fibrose die \\ Unterscheidung der allergischen bronchopulmonalen Aspergillose \\ von einer unkomplizierten Aspergillus-Allergie erlaubt.
}

Die allergische bronchopulmonale Aspergillose (ABPA) ist eine relativ häufige Komplikation bei Patienten mit zystischer Fibrose (CF). Die Prävalenz liegt bei 6 bis $10 \%$. Bislang mangelte es jedoch an einem spezifischen Nachweis der ABPA, da jeder vierte CF-Patient eine Allergie gegen Aspergillus fumigatus aufweist. Sowohl die ABPA als auch die unkomplizierte Allergie gehen mit erhöhten IgE-Spiegeln gegen A. fumigatus einher, lassen sich dadurch also nicht unterscheiden. Die Sensitivität erhöhter Aspergillus-IgE-Titer liegt zwar bei $92 \%$, die Spezifität aber nur bei $58 \%$. Daher haben Sally Anne Little und John Oliver Warner von der Universitätsklinik Southhampton nach Antigenen gesucht, die eine spezifischere Diagnostik erlauben.

\section{Spezifisches Protein für allergische} bronchopulmonale Aspergillose: gp66

Serumproben von neun CF-Patienten mit allergischer bronchopulmonaler Aspergillose sowie von fünf Patienten mit unkomplizierter Allergie gegen Aspergillus fumigatus wurden mit aufwendigen immunologischen Analysemethoden auf das Vorhandensein von Aspergillus-fumigatusProteinen untersucht. Das Ziel war, Proteine zu finden, die zum einen spezifische IgE-Antworten erzeugen, zum anderen möglichst nur bei der ABPA nachzuweisen sind. Als Methode zum Protein-Screening bedienten sich die Wissenschaftler der gekreuzten Radioimmuno-Elekrophorese. Die Molukulargewichte wurden mittels Elektrophorese und Immunoblotting bestimmt.

Die Suche war erfolgreich: Tatsächlich wurde ein Allergen gefunden, das offenbar überwiegend bei der allergischen bronchopulmonalen Aspergillose in Erscheinung tritt. Es handelt sich um ein Glykoprotein mit einem Molekulargewicht von 66 Kilodalton, weshalb es den vorläufigen Namen gp66 erhielt.

\section{Spezifität auf knapp $80 \%$ erhöht}

Nach Identifikation des Proteins wurde ein ELISA zum Nachweis von spezifischen IgE-Antikörpern gegen gp66 entwickelt. Um die Effektivität dieses ELISA zu bestimmen, verglichen die britischen Forscher das Ergebnis mit dem der herkömmlichen IgE-Bestimmung, bei der unfraktionierte Aspergillus-fumigatus-Extrakte als Probenmaterial verwandt werden. Die Proben dieser Vergleichsstudie stammten von 14 Patienten mit zystischer Fibrose und allergischer bronchopulmonaler Aspergillose, 42 Patienten mit zystischer Fibrose ohne ABPA, aber einer Aspergillus-Allergie, sowie 23 Patienten mit zystischer Fibrose ohne ABPA und Allergie.

Die Bestimmung von gp66 erhöhte die Spezifität des ABPA-Nachweises von $58,5 \%$ auf $78,5 \%$, während die Sensitivität unverändert bei $92,9 \%$ verblieb. Für den Nachweis von gp66 scheint sich dabei ELISA besser als RAST zu eignen.

Nach Ansicht der Autoren besteht damit erstmals die Möglichkeit der zuverlässigen serologischen Diagnose einer allergischen bronchopulmonalen Aspergillose, und zwar möglicherweise schon in den frühesten Krankheitsstadien.

(zo)

Quelle: Little SA, Warner JO: Improved diagnosis of allergic bronchopulmonary aspergillosis with gp66 (formerly antigen 7) of Aspergillus fumigatus for specific IgE detection. J. Allergy Clin. Immunol. (1996) 98: 55-63. 